\title{
Conhecimento de gestantes sobre a fisioterapia pélvica
}

\section{Level of knowledge of pregnant women about pelvic physioterapy}

\author{
Kátia Regina Strutz ${ }^{1}$, Marlise Uber ${ }^{1}$, Viviane Bolfe Azzi², Erica Feio Carneiro Nunes ${ }^{3}$, Gustavo \\ Fernando Sutter Latorre ${ }^{4}$ \\ 1 - Faculdade Inspirar, Curitiba, PR, Brasil. \\ 2 - Universidade do Oeste de Santa Catarina, Campus São Miguel do Oeste, SC, Brasil. \\ 3 - Universidade do Estado do Pará, Belém, PR, Brasil. \\ 4 - Fundación Univeristária Iberoamericana - FUNIBER, Paseo García Faria, Espanha.
}

\section{RESUMO}

erica@perineo.net

Introdução: a pelve humana é responsável pela função urinária, fecal, sexual e obstétrica. A presença do fisioterapeuta pélvico ainda não é uma realidade no sistema de saúde para muitas cidades, mesmo sendo reconhecida pela Organização Mundial de Saúde como importante no acompanhamento gestacional e no parto. Objetivo: verificar o conhecimento de gestantes a respeito da fisioterapia pélvica. Método: gestantes maiores de 18 anos que participam de grupos de apoio local responderam um questionário sociodemográfico com questões abertas e fechadas. Dados foram categorizados e analisados descritivamente. A análise qualitativa foi segundo critério de recorrência dos significados. Resultados: vinte e oito mulheres responderam ao questionário, sendo 75\% com idade entre 23 a 34 anos, 74\% com escolaridade em ensino superior ou pós-graduação e $68 \%$ primigestas; $78 \%$ ouviram falar em fisioterapia pélvica, $22 \%$ passaram por algum tipo de atendimento durante a gestação

Palavras-chave: e todas as entrevistadas indicariam o serviço para outras gestantes. Conclusão: apesar de a Fisioterapia A ssoalho maioria das gestantes conhecerem a fisioterapia pélvica, poucas realizaram o atendimento, pélvico Gravidez Saúde apontando como principal fator o não oferecimento da fisioterapia pélvica no sistema púPública blico de saúde.

\begin{abstract}
Introduction: the human pelvis is responsible for urinary, fecal, sexual and obstetric functions. The presence of a pelvic physiotherapist is not yet a reality in the public health system for many cities, even though it is recognized by the World Health Organization as fundamental during pregnancy and delivery. Objective: to verify the knowledge of pregnant women regarding pelvic physiotherapy. Method: pregnant women over 18 years old, who participate in local support groups, answered a sociodemographic questionnaire with open and closed questions. Data were categorized and analyzed descriptively. Qualitative analysis was based on the criterion of recurrence of meanings. Results: twenty-eight women answered the questionnaire, $75 \%$ aged $23-34$ years, $74 \%$ with higher education or post-graduation, and $68 \%$ were primigravidae; $78 \%$ heard about pelvic physiotherapy, $22 \%$ went through

Keywords: some type of care during pregnancy and all the interviewees indicated the service to other Physiotherapy; pregnant women. Conclusion: although most pregnant women were aware of pelvic physio-
\end{abstract} Pregnancy; Pelvic Floor; therapy, and wanted to do it, few performed the care. The main factor related was the failure Public Health. to provide pelvic physiotherapy in the public health system. 


\section{INTRODUÇÃO}

A pelve humana consiste num arcabouço formado pelos ossos ílio, ísquio, púbis, sacro e cóccix e se localiza através de duas bases: a maior, em que se encontram as vísceras e a menor, que é a continuação mais estreita da pelve maior. A abertura pélvica inferior é fechada por um conjunto de tecidos moles conhecido como assoalho pélvico. Este é composto de camadas de músculos e fáscias, com as funções de suporte para as vísceras, resistência ao aumento da pressão intra-abdominal e ação esfincteriana da uretra, vagina e reto. ${ }^{1}$ A sustentação do peso do bebê durante a gestação e o auxílio na sua expulsão durante o parto também são funções atribuídas ao assoalho pélvico. ${ }^{2}$

$\mathrm{Na}$ mulher, a pelve possui a abertura superior mais larga para facilitar o encaixe e a passagem do bebê durante o parto e a inferior também é mais larga, o que predispõe ao enfraquecimento da musculatura do assoalho pélvico, podendo sofrer rupturas e desencadear prolapsos e incontinência urinária e/ou fecal. ${ }^{1}$ Segundo Marcelino e Lanuez, ${ }^{3}$ a lesão perineal afeta $85 \%$ das que realizam parto vaginal.

Mecanicamente, durante o parto é necessário que os ossos pélvicos se mobilizem, aumentando progressivamente o canal ósseo de parto e permitindo que o bebê chegue até o estreito inferior, de onde o assoalho pélvico deve ceder para permitir a passagem no final do período expulsivo. A movimentação destes ossos acontece por meio das articulações sacro-ilíacas, cuja mobilidade deve ser suficiente para uma abertura eficaz dos estreitos ósseos, enquanto o assoalho pélvico deve estar maleável o suficiente para permitir uma passagem sem roturas teciduais. ${ }^{4,5}$ Tanto a mobilidade das articulações sacro-ilíacas quanto a maleabilidade do assoalho pélvico podem ser treinadas ou tratadas por meio da fisioterapia pélvica.

Desde 2005, a fisioterapia pélvica é reconhecida como padrão ouro para o tratamento de disfunções do assoalho pélvico como a incontinência urinária, tendo importância crescente no tratamento de outras disfunções como disfunções anorretais, sexuais e prolapsos genitais. ${ }^{6} \mathrm{O}$ fisioterapeuta pélvico pode atuar na preparação da mulher para as mudanças físicas que ocorrem na gravidez e puerpério, minimizando o estresse e orientando posicionamentos biomecanicamente favoráveis para o momento do parto, além do relaxamento efetivo da musculatura e respiração adequada. No pós-parto, orientará exercícios específicos para essa fase e, se necessário, um tratamento especializado para o tratamento de disfunções. $^{3}$

A assistência profissional atual, muitas vezes é organizada em função das necessidades das instituições e não das parturientes. Atitudes e procedimentos que priorizem a qualidade da atenção prestada são fundamentais nesse período, afim de ultrapassar o modelo centrado apenas no monitoramento e controle de risco. ${ }^{7} \mathrm{O}$ parto é entendido como um evento biopsicossocial, permitindo assim a participação ativa da mulher na escolha dos procedimentos, favorecendo a promoção da saúde, proteção ao nascimento e os aspectos emocionais. ${ }^{8} \mathrm{~A}$ intervenção fisioterapêutica na assistência obstétrica como parte da rotina, valoriza o protagonismo da gestante, que utiliza o movimento ativo do próprio corpo como uma ferramenta para facilitar o processo de trabalho de parto e trazer satisfação com o nascimento. O fisioterapeuta como um profissional do movimento, tem conhecimento para auxiliar nesse processo. ${ }^{9}$

A presença do fisioterapeuta no acompanhamento do trabalho de parto não é uma prática estabelecida e incluída no Sistema Único de Saúde (SUS) brasileiro. Geralmente as experiências encontradas são em clínicas privadas ou em maternidade-escola. A atuação do fisioterapeuta, tanto no sistema público, quanto no privado, é voltada principalmente para a atenção pré-natal, sem sua presença no hospital. Na Europa, é uma prática comum encontrar fisioterapeutas atuando tanto na preparação pré-natal, como na assistência ao parto, estando incluídos na equipe obstétrica. ${ }^{7}$

Todavia, mesmo sendo reconhecida pela Organização Mundial da Saúde (OMS) como importante na condução do parto, a fisioterapia pélvica ainda não é realidade no sistema público de saúde brasileiro. Uma das estratégias para implementar novos programas de saúde na rede pública é fomentar o conhecimento público a partir de campanhas de esclarecimento popular, mas, para tanto, é necessário que antes, se tenha um conhecimento panorâmico do estado atual do conhecimento popular a respeito da fisioterapia pélvica, neste caso, especificamente de gestantes. Assim, o objetivo do presente estudo, que compõe uma série de estudos projetando implantar um serviço de fisioterapia pélvica para gestação e parto no município de Blumenau - SC, foi verificar o conhecimento de gestantes a respeito da fisioterapia pélvica. 


\section{MÉTODO}

Trata-se de um estudo transversal, aprovado no Comitê de Ética em Pesquisa Científica da Faculdade Inspirar (parecer número 2.608.013), realizado no município de Blumenau, Santa Catarina, no período de maio a junho de 2018. A amostra do estudo foi composta por gestantes que participavam de grupos de apoio às gestantes oferecidos por doulas e enfermeiras que atuavam em Unidade Básicas de Saúde do município. Como critérios de inclusão, foram selecionadas gestantes em qualquer período gestacional, maiores de 18 anos e com endereço eletrônico (e-mail) ativo e de exclusão gestantes de risco.

Aos responsáveis pelos grupos de apoio às gestantes foi explicado o objetivo, bem como os critérios de elegibilidade do estudo, para que os mesmos encaminhassem as gestantes que quisessem participar. Para as que aceitavam, o responsável pelo grupo anotava oe-mail da participante. Foi estabelecido contato via e-mail com cada gestante, apresentando o projeto e sanando possíveis dúvidas. Então, era encaminhado o link da plataforma Formulários Google, contendo o Termo de Consentimento Livre e Esclarecido e o questionário, que foi preenchido de forma anônima, sendo visualizado unicamente pelos pesquisadores.

$\mathrm{O}$ instrumento utilizado para a pesquisa foi um questionário sociodemográfico, elaborado pelos autores, composto por doze questões mistas, sendo cinco abertas e sete fechadas que contemplam o delineamento do perfil pessoal e questões relativas à atuação da fisioterapia pélvica. Os dados coletados foram armazenados em um banco de dados no programa Microsoft Excel $^{\circledR}$. As informações foram categorizadas e as variáveis analisadas descritivamente por meio de frequência absoluta e relativa. Para a análise qualitativa, realizou-se a leitura dos dados, separando e organizando segundo critério de recorrência dos significados.

\section{RESULTADOS}

A amostra total foi composta por vinte e oito mulheres que aceitaram participar deste estudo. $\mathrm{Na}$ tabela 1, estão apresentadas as variáveis referentes ao perfil da gestante entrevistada.

Tabela 1 - Perfil das participantes.

\begin{tabular}{lc}
\hline \multicolumn{1}{c}{ Variáveis } & $\mathbf{n}(\%)$ \\
\hline Idade (anos) & $11(39,3)$ \\
$23-28$ & $10(35,7)$ \\
$29-34$ & $7(25,1)$ \\
$35>$ & \\
Grau de escolaridade & $1(3,7)$ \\
Ensino Fundamental & $4(14,8)$ \\
Ensino Médio & $9(33,3)$ \\
Graduação & $11(40,7)$ \\
Pós-graduação & $2(7,4)$ \\
Outros & \\
Número de gestações & $19(67,9)$ \\
1 & $9(32,1)$ \\
2 & \\
Gestações anteriores & $7(25,9)$ \\
Parto normal & $3(11,1)$ \\
Cesárea & $17(63)$ \\
Não respondido &
\end{tabular}

Tabela 2 - Sobre a atuação da fisioterapia pélvica.

\begin{tabular}{lc}
\hline \multicolumn{1}{c}{ Variáveis } & $\mathbf{n}(\%)$ \\
\hline Conhecimento da fisioterapia pélvica & $22(78,6)$ \\
Sim & $6(21,4)$ \\
Não & $12(42,9)$ \\
Conheceram através de quem? & $6(21,4)$ \\
Profissionais da saúde & $4(14,2)$ \\
Amigos & $6(21,4)$ \\
Internet & $5(17,8)$ \\
Outros & $23(82,2)$ \\
Realizaram atendimento de fisioterapia pélvica & $5(17,8)$ \\
Sim & $23(82,2)$ \\
Não & $5(17,8)$ \\
Consideram importante a atuação da fisioterapia pélvica & $23(82,2)$ \\
Sim & 50 \\
Não se aplica & \\
Indicam o serviço para outras gestantes & \\
Sim & \\
Não se aplica & \\
\hline
\end{tabular}


Os dados da tabela 2, envolvem questões referentes à atuação da fisioterapia pélvica. Quando questionadas se conhecem a fisioterapia pélvica, $78,6 \%(n=22)$ das entrevistadas responderam que sim. Das vinte e duas que conhecem a atuação do fisioterapeuta pélvico, $42,9 \%(\mathrm{n}=12)$ procuraram o serviço por indicação de profissionais da saúde, $21,4 \%$ $(n=6)$ por indicação de algum amigo, 14,2\% $(n=4)$ através da internet e $21,4 \%(n=6)$ por outros meios conheceram a atuação na graduação, por sugestão da instrutora de Pilates e no curso de gestantes.

Quando questionadas se realizaram atendimento de fisioterapia pélvica, 82,2\% $(n=23)$ responderam que não. Todas as gestantes que realizam o acompanhamento fisioterapêutico especializado consideram importante e indicam o serviço para outra gestante. Destaca-se como recorrência de significados nas falas das gestantes, as seguintes abordagens: "sim e já indico", "sim, muitas amigas fazem também", "sempre indico", o que também demonstra a satisfação com o serviço.

\section{DISCUSSÃO}

O presente estudo teve por objetivo verificar o conhecimento das gestantes acerca da fisioterapia pélvica e revelou que apesar das participantes avaliadas do estudo conhecerem a fisioterapia pélvica, poucas passaram por algum tipo de atendimento durante a gestação. As participantes que tiveram acompanhamento da fisioterapia pélvica durante a gestação consideram importante a atuação do fisioterapeuta pélvico e indicam o serviço as outras gestantes, sendo este um indicativo do desejo destas mulheres por este serviço durante a preparação para o parto e pós-parto.

Caixeta Neto et al., ${ }^{10}$ realizaram um estudo com 80 mulheres ( 40 gestantes e 40 puérperas), com idades entre 16 e 38 anos, selecionadas aleatoriamente, para avaliar o conhecimento a respeito do tratamento fisioterapêutico na incontinência urinária de esforço entre gestantes e puérperas atendidas na rede pública de saúde do município de Patrocínio-MG. Tais autores observaram baixo conhecimento sobre a atuação da fisioterapia no tratamento da incontinência urinária de esforço, evidenciando a necessidade de ampliação da assistência fisioterapêutica ginecológica e obstétrica nos serviços públicos, além da urgência na implementação de atividades de educação em saúde da mulher.
Embora o estudo de Caixeta Neto et al. ${ }^{10}$ tenha sido relacionado à incontinência urinária de esforço, ele demonstra uma falta de conhecimento sobre as possibilidades de atuação da fisioterapia pélvica e falta de oferta deste serviço na rede pública de saúde. É provável que o implemento de um serviço de fisioterapia pélvica no serviço público aumente consideravelmente o atendimento às gestantes neste sentido, facilitando a adaptação as mudanças corporais ocorridas durante a gestação e durante o parto, além de minimizar as sequelas no pós-parto.

No Brasil, o modelo de atenção às gestantes vem sendo discutido e construído para assegurar a melhoria do acesso, da cobertura e da qualidade do acompanhamento pré-natal, da assistência ao parto e puerpério às gestantes e ao recém-nascido. Dentre os serviços oferecidos pelo SUS percebe-se uma lacuna no que diz respeito à fisioterapia pélvica. Apesar de em 2017 ter sido lançado, pelo Ministério da Saúde, o documento das Diretrizes Nacionais de Assistência ao Parto Normal ${ }^{11}$ o mesmo não menciona a fisioterapia pélvica como um recurso a ser utilizado durante o período gestacional, muito embora haja, atualmente, forte evidência a respeito desta terapia na prevenção e tratamento das disfunções do assoalho pélvico relacionadas à gestação e ao parto. ${ }^{6,12-14} \mathrm{Na}$ mesma linha, pode-se também citar a Caderneta de Gestante, ${ }^{15}$ presente durante as rotinas pré-natais, na qual constam informações importantes para este período, mas que, novamente, não aborda a importância da preparação pélvica para o parto ou para a prevenção/recuperação das possíveis sequelas no pós-parto, quando for o caso.

Além das medidas educativas que devem ser introduzidas nos programas de pré-natal durante a gravidez, a Cartilha Parto, Aborto e Puerpério ${ }^{15}$ enfatiza que a preparação da mulher para o nascimento compreende principalmente a adoção de medidas referentes ao trabalho corporal, com o objetivo de oferecer à mulher um melhor conhecimento da percepção do corpo, bem como do relaxamento e da respiração para melhor controle do trabalho de parto e parto, e para isso, é necessário trabalhar com a gestante exercícios próprios para cada etapa da gravidez. ${ }^{16}$ Todavia, durante o trabalho de parto, a gestante necessita de mobilidade pélvica e o uso da musculatura do abdômen, assoalho pélvico e diafragma respiratório, ${ }^{17}$ musculaturas que devem ser treinadas especificamente. Particularmente o assoalho pélvico é uma região difícil de identificar, a qual 
um terço das mulheres simplesmente não consegue contrair por conta própria, sem antes ser instruída por um fisioterapeuta pélvico especializado. ${ }^{18}$ Mesmo depois de aprender a contrair, os exercícios de reforço desta musculatura devem seguir uma ordem baseada na aprendizagem motora, que deva conter, nesta ordem, um programa de propriocepção, seguido de um de coordenação motora, para finalmente ser realizado o programa de reforço que deve ser finalizado por um programa de treinamento funcional específico, baseado nas atividades de vida diária específicas de cada indivíduo. ${ }^{6}$ Neste ínterim, a fisioterapia se faz, mais do que necessária, fundamental.

Ofisioterapeutaé o profissional capacitadopara atuar com o movimento do corpo humano, portanto, auxilia na contração e relaxamento da musculatura, trabalhando com o intuito de otimizar a fisiologia humana. Também é importante na preparação do assoalho pélvico durante a gravidez e reeducação no pós-parto e, para este fim, existe a especialidade em fisioterapia pélvica. ${ }^{19}$ No atendimento a gestante, a fisioterapia tem como objetivo a preparação para um parto tranquilo e a prevenção de complicações, proporcionando uma melhor qualidade de vida. ${ }^{17}$

Dentro dessa perspectiva, com a implantação do serviço no setor público, o acesso a esses tratamentos seria facilitado, visto que atualmente estão à disposição da rede privada ou convênios de saúde. Devido à grande morbidade associada aos custos elevados e à alta prevalência, considera-se a incontinência urinária (IU) um problema de saúde pública. $^{20}$

A fisioterapia pélvica é indicada na preparação da gestante para o parto, com custo financeiro menor quando comparado aos gastos com procedimentos cirúrgicos, além de oferecer baixo risco e efeitos colaterais. $^{20,21}$

Como limitações deste estudo citamos o número amostral pequeno e o fato de ter sido realizado de forma on-line, o que não permitiu a participação de gestantes que não tivessem acesso à internet.

\section{CONCLUSÃO}

As participantes deste estudo tinham conhecimento acerca da Fisioterapia Pélvica, porém somente poucas passaram por algum tipo de atendimento fisioterapêutico durante a gestação. A ausência desse tipo de serviço na rede pública de saúde pode ser a causadora desta escassez de assistência fisioterapêutica para gravidez e preparação para o parto, visto que esses atendimentos atualmente estão disponíveis na rede privada e convênios de saúde.

No Brasil, vem sendo discutido e construído um modelo de atenção as gestantes, para garantir um atendimento de qualidade a assistência humanizada ao parto e puerpério, mas percebe-se uma lacuna no que diz respeito a atuação da fisioterapia pélvica, pois entre os serviços oferecidos, não citam como opção de tratamento.

Com a presença da fisioterapia pélvica no serviço público, mais mulheres teriam acesso ao tratamento conservador, reduzindo o número de procedimento cirúrgicos, em consequência a redução de custos públicos e das próprias pacientes, possibilitando maior adesão ao tratamento e diminuindo a fila de espera.

\section{REFERÊNCIAS}

1. Palma PCR, Berghmans B, Seleme MR, Riccetto CLZ, Pereira SB. Urofisioterapia. Aplicação clínica das Técnicas Fisioterapêuticas nas disfunções Miccionais e do Assoalho Pélvico. 2.ed. São Paulo: Manole, 2014. 524 p.

2. Marques A, Ponzio MPS, Pace do Amaral MT. Tratado de Fisioterapia em Saúde da Mulher. São Paulo: Editora Roca LTDA, 2011. $455 \mathrm{p}$.

3. Marcelino TC, Lanuez FV. Abordagem fisioterapêutica na massagem perineal no pré-parto. ConScientia e Saúde 2009;8(2):339-44.

4. Gasquet B. Trouver sa position d'accouchement. Paris: Marabout, 1999. 126 p.

5. Gasquet B. Périnée arrêtons le massacre!: Prévenir et réparer les erreurs répétées dans la vie quotidienne, la maternité, le sport. Paris: Marabout, 2011. 224 p.

6. Bø K, Berghmans B, Mørkved S, Van Kampen M. EvidenceBased Physical Therapy for the Pelvic Floor: Bridging Science and Clinical Practice. 2 ed. London: Churchill Livingstone, 2015. $446 \mathrm{p}$.

7. Bio E. Intervenção fisioterapêutica na assistência ao trabalho de parto [dissertação]. São Paulo: Faculdade de Medicina da Universidade de São Paulo; 2007.

8. Domingues R, Santos E, Leal M. Aspectos da satisfação das mulheres com a assistência ao parto: contribuição para o debate. Cad Saude Publica 2004; 20(Suppl 1):52-S62. doi: http://dx.doi. org/10.1590/S0102-311X2004000700006

9. Bavaresco GZ, Souza R, Almeica B, Sabatino J, Dias M. $\mathrm{O}$ fisioterapeuta como profissional de suporte à parturiente. Cien Saude Colet 2011;16(7):3259-66. doi: http://dx.doi. org/10.1590/S1413-81232011000800025

10. Caixeta Neto AG, Gonçalves AC, Ribas LF, Silva JAF. Avaliação do conhecimento sobre o tratamento fisioterápico da incontinência urinária de esforço entre gestantes e puérperas atendidas na rede pública de saúde do município de PatrocínioMG. Rev. Interdisciplin. Promoç. Saúde 2018;1(1): 41-5. doi: 
http://dx.doi.org/10.17058/rips.v1i1.11942

11. Brasil. Ministério da Saúde. Secretaria de Ciência, Tecnologia e Insumos Estratégicos. Departamento de Gestão e Incorporação de Tecnologias em Saúde. Diretrizes nacionais de assistência ao parto normal: versão resumida. Brasília: Ministério da Saúde; 2017.

12. Bø K, A H Haakstad L, Voldner N. Do pregnant women exercise their pelvic floor muscles? IntUrogynecol J Pelvic Floor Dysfunct 2007;18(7):733-6. doi: http://dx.doi.org/10.1007/ s00192-006-0235-2

13. Hay-Smith J, Mørkved S, Fairbrother KA, Herbison GP. Pelvic floor muscle training for prevention and treatment of urinary and faecal incontinence in antenatal and postnatal women. Cochrane Database Syst Rev 2008;8(4):CD007471. doi: http://dx.doi.org/10.1002/14651858.CD007471.pub4 14. Mørkved S, Bø K, Schei B, Salvesen KA. Pelvic Floor Muscle Training During Pregnancy to Prevent Urinary Incontinence: A Single-Blind Randomizes Controlled Trial. American College of Obstetricians and Gynecologists 2002;100(1):72-8. doi: http:// dx.doi.org/10.1016/s0029-7844(02)02711-4

15. Brasil. Ministério da Saúde. Secretaria de Políticos de Saúde. Área Técnica de Saúde da Mulher. Parto, aborto e puerpério: assistência humanizada à mulher. Brasília: Ministério da Saúde; 2001.

16. Souza A, Ramos D. Fisioterapia e Humanização do Parto: uma análise partir de documentos oficiais da saúde. R. fisioter. reab 2017;1(1):11-23.

17. Canesin K, Amaral W. Atuação fisioterapêutica para diminuição do tempo do trabalho de parto: revisão de literatura. Femina 2010;38(8):429-33.

18. Henderson JW, Wang S, Egger MJ, Masters M, Nygaard I. Can women correctly contract their pelvic floor muscles without formal instruction? Female Pelvic Med Reconstr Surg 2013;19(1): 8-12. doi: http://dx.doi.org/10.1097/SPV.0b013e31827ab9d0

19. Associação Brasileira de Fisioterapia Pélvica (ABFP). O que é fisioterapia pélvica [documento na internet]. Florianópolis: Associação Brasileira de Fisioterapia Pélvica; 2018 [citado em 15 de outubro de 2018]. Disponível em: http://abfp.org.br

20. Vaz, C. Assistência Fisioterapêutica a mulheres com incontinência urinária na atenção básica. [Dissertação de Mestrado]. Minas Gerais: Universidade Federal de Minas Gerais; 2012.

21. Figueiredo EM, Lara JO, Cruz MC, Quintão DMG, Monteiro MVC. Perfil sociodemográfico e clínico de usuárias de serviço de Fisioterapia Uroginecológica da rede pública. Rev Bras Fisioter 2008;12(2):136-42. doi: http://dx.doi.org/10.1590/ S1413-35552008000200010

Como citar: STRUTZ, Kátia Regina et al. Conhecimento de gestantes sobre a fisioterapia pélvica. Revista Interdisciplinar de Promoção da Saúde, Santa Cruz do Sul, v. 2, n. 4, out. 2019. ISSN 2595-3664. Disponível em: $<$ https://online.unisc.br/seer/index. php/ripsunisc/article/view/15126>. Acesso em: 01 out. 2019. doi:https://doi.org/10.17058/rips.v2i4.15126 